\title{
O uso da caneta injetora de insulina no cotidiano: percepções do adolescente
}

\author{
The insulin pen usage on daily life: adolescent perceptions
}

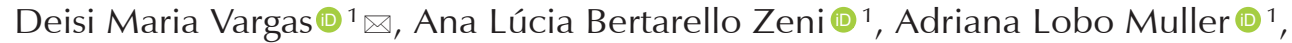 \\ Claudia Regina Lima Duarte da Silva ${ }^{1} 1$ \\ ${ }^{1}$ Universidade Regional de Blumenau (FURB). Blumenau, SC, Brasil. \\ Fontes de financiamento: Bolsa de estudo PIPe/artigo 170 (Estado de Santa Catarina) e Dispositivos caneta uma doação do Laboratório Lilly.
}

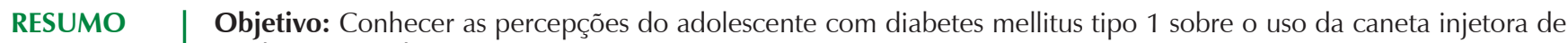
insulina no cotidiano.

Materiais e Métodos: Realizou-se uma pesquisa qualitativa envolvendo 15 adolescentes com diabetes inscritos em um programa universitário de extensão, com 13 a 18 anos de idade. Os jovens do sexo masculino e feminino com assiduidade de no mínimo três reuniões por ano nas atividades do grupo foram convidados a participar do estudo. As entrevistas semiestruturadas foram desenvolvidas antes e depois do uso contínuo da caneta aplicadora de insulina. Os dados foram interpretados através da análise de conteúdo proposta por Bardin. O projeto foi aprovado pelo comitê de ética da Universidade Regional de Blumenau sob o parecer número 99/10.

Resultados: Nove adolescentes com diabetes, sendo quatro do sexo feminino e cinco do sexo masculino revelaram que se sentem impotentes frente à mudança causada pela doença e a consequente perda de controle sobre a própria vida. Além disso, a condição de ter diabetes e aplicar a insulina com seringa e agulha foi apontada como uma das dificuldades do tratamento. Sobre a caneta, todos os adolescentes responderam que a preferem devido à praticidade no desenvolvimento das atividades cotidianas.

Conclusão: Neste estudo foi percebido que o uso da caneta injetora de insulina contribui para a melhoria da qualidade de vida, merecendo mais atenção dos profissionais e da gestão pública em saúde visando o estímulo ao autocuidado.

Palavras-chave: saúde do adolescente; diabetes mellitus; qualidade de vida; insulina.

\begin{tabular}{l|l} 
ABSTRACT & Objective: To know the perceptions of diabetes mellitus type 1adolescents on the use of the insulin injection pen in daily life.
\end{tabular}

Materials and Methods: A qualitative research was carried out involving fifteen diabetic adolescents aged 13 to 18 years-old enrolled in a university extension program. The young men and women attending at least three meetings a year in group activities were invited to participate in the study. Semi-structured interviews were performed before and after the routine use of the insulin pen. The data were analyzed and interpreted through content analysis proposed by Bardin. The ethics committee of FURB approved the project under the protocol number 99/10.

Results: Nine adolescents with diabetes, four female and five male, revealed that they feel helpless in the face of the changes caused by the disease and the consequent loss of control over their lives. In addition, the condition of having diabetes and needing to apply insulin with a syringe and needle was pointed out as one of the difficulties of the treatment. Regarding the pen, all adolescents prefer it considering the practicality in the development of daily activities.

Conclusion: In this study, it was perceived that the use of the insulin injection pen contributes to the improvement on the quality of life of the adolescent with diabetes, deserving more attention by professionals and health public management aiming to promote self-care.

Keywords: adolescent health; diabetes mellitus; quality of life; insulin.

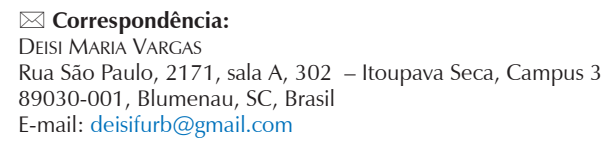




\section{INTRODUÇÃO}

Diabetes mellitus tipo 1 (DM1) é uma das doenças crônicas mais prevalentes em adolescentes. A cada ano cerca de 70.000 crianças e adolescentes são diagnosticadas com DM1 no mundo, com crescimento anual de 5\% na idade pré-escolar e 3\% na adolescência ${ }^{1,2}$. O tratamento e o controle metabólico da DM1 podem comprometer a qualidade de vida do adolescente já que exigem alterações de comportamento como mudanças nos hábitos alimentares e no estilo de vida, bem como o uso de medicação injetável contínua ${ }^{3}$.

No contexto da adolescência, a convivência com uma doença crônica se torna ainda mais complicada comparando com um adulto, pois afeta o cotidiano podendo causar ruptura com o meio social ${ }^{4}$. O ritmo de vida do adolescente é alterado, pois alguns aspectos do tratamento o impedem de realizar atividades comuns. O jovem com doença crônica pode ver sua perspectiva de conquista de autonomia e identidade bloqueadas pelos hábitos diários de autocuidado, assim como regras alimentares que escapam em muito ao perfil comum dos seus pares 5 . O senso de pertencimento a um grupo é essencial no desenvolvimento da identidade do adolescente, pois é nesta fase que práticas, momentos e acontecimentos triviais bem como o lazer adquirem importância na vida em sociedade ${ }^{4}$. Assim, as mudanças de cotidiano impostas pela doença podem gerar rejeição ao tratamento com prejuízos à sobrevida, à convivência social, e consequentemente à constituição do sujeito social ${ }^{5}$. Além disto, há o estigma de ser acometido por uma doença crônica, ter a necessidade de submeter-se a procedimentos em lugares públicos e correr o risco da ocorrência de hipoglicemia, reforçam a condição de ser diferente podendo gerar sentimentos de inferioridade e rebeldia ${ }^{2,6}$.

O adolescente com DM1 precisa de várias aplicações de insulina durante o dia em diferentes situações do cotidiano como, escola e trabalho entre outras atividades fora de casa. Assim, transportar e aplicar seu medicamento de forma segura e prática, sem expor publicamente sua condição diferente e sem assumir riscos de contaminação ou má conservação do medicamento, seria mais adequado para estes adolescentes.

A autoaplicação da insulina é um importante passo para a autonomia do adolescente com diabetes, mas o medo da agulha, aliado às crenças errôneas sobre a insulina, acabam por afastar a motivação em autoaplicar, reforçando a postura de dependência do diabético a outra pessoa ${ }^{7}$. Além disso, a administração de insulina requer cuidados específicos, quanto ao local de preparo, de transporte e armazenamento. Por outro lado, o uso da caneta injetora além de melhorar a autoaplicação apresenta vantagens como, facilidade de preparo, armazenamento e transporte, uma vez que não é necessário refrigeração e, redução do risco de contaminação, pois o envase da insulina vem pronto para uso.

Neste sentido, o impacto do tratamento do DM1 não ocorre apenas na esfera biológica, mas permeia também a esfera psicossocial, surgindo a necessidade de estabelecer ações que permitam conduzir a vida cotidiana dentro de pressupostos aceitáveis pelo adolescente com diabetes, incentivando o uso de tecnologias leve-duras, no caso unindo autonomização e vínculos com as necessidades de ações na saúde com os equipamentos ${ }^{8}$. Conforme Merhy et al. ${ }^{8}$ não há um modo único de realizar procedimentos, podendo equilibrar a dureza e a leveza, trabalhado-se na tentativa de compreender a situação e como enfrentar, utilizando práticas de saúde que os tornem mais autônomos para ampliar as escolhas sobre as atividades desenvolvidas junto aos seus pares. Desta forma, desenvolveu-se este estudo com o objetivo de conhecer as percepções do adolescente com DM1 sobre o uso da caneta injetora de insulina no cotidiano.

\section{MATERIAIS E MÉTODOS}

Trata-se de uma pesquisa qualitativa realizada em um ambulatório universitário que integra a rede de serviços do Sistema Único de Saúde (SUS), cenário de prática de um programa de extensão universitária em educação em saúde com foco em atenção integral voltado a crianças e adolescentes com diabetes. Uma das atividades do programa são reuniões mensais voltadas às necessidades de saúde dos sujeitos do cuidado, seus familiares e cuidadores. O universo para ao estudo era de 15 adolescentes com 13 a 18 anos com diagnóstico de DM1 há no mínimo três e no máximo dez anos, dentro dos critérios estabelecidos pela Sociedade Brasileira de Diabetes. Os adolescentes com assiduidade de no mínimo três reuniões por ano nas atividades do grupo foram convidados a participar do estudo seguindo o método de amostragem por saturação ${ }^{9}$.

A pesquisa foi dividida em dois momentos distintos, o primeiro compreendeu a realização de entrevista semiestruturada através de questões abertas direcionadas à caracterização da qualidade de vida com o uso da insulina. Após a entrevista, as canetas para a aplicação de insulina foram distribuídas aos participantes da pesquisa. Cada participante recebeu duas canetas, uma para a aplicação da insulina de ação intermediária (NPH) e outra para a aplicação da insulina de ação rápida (regular). A orientação sobre o funcionamento do dispositivo e as rotinas decorrentes da alteração no método de aplicação da insulina foi realizada por uma equipe interprofissional e a nova rotina foi acompanhada pelo médico responsável. 
O segundo momento compreendeu a realização de outra entrevista semiestruturada após o uso do dispositivo tipo caneta pelos sujeitos da pesquisa. O tempo de uso foi de 6 meses no mínimo e o máximo foi dependente do retorno para avaliação médica (até 16 meses). Nesta entrevista, foram investigados aspectos relacionados a autonomia, transporte, experiências de aplicação bem como aspectos de qualidade de vida. Todas as entrevistas foram gravadas em arquivo digital e, posteriormente transcritas, preservando a veracidade dos depoimentos obtidos.

Para a análise e interpretação dos dados, utilizou-se a análise de conteúdo segundo Bardin ${ }^{10}$ composta por três etapas, pré-análise, exploração do material e tratamento de resultados. Foram utilizadas categorias reflexivas adolescência, convívio social, controle da glicemia e autonomia.

Este estudo foi aprovado pelo Comitê de Ética e Pesquisa com Seres Humanos da Universidade Regional de Blumenau sob o número 99/10.

\section{RESULTADOS}

Conforme critérios de seleção descritos na metodologia resultaram nove adolescentes participantes deste estudo sendo quatro do sexo feminino e cinco do sexo masculino. A seguir os resultados estão organizados conforme as categorias analisadas.

\section{O olhar do adolescente com diabetes sobre sua condição de vida e saúde}

A partir do diagnóstico do DM1, os adolescentes experimentam a ideia de mudança radical e perda de controle sobre sua vida. $O$ adolescente sente-se na obrigação de adaptar-se à sua nova rotina, que muitas vezes é simplesmente imposta, gerando uma sensação de impotência e deixando-o com a sensação de espectador da própria vida, não podendo mais controlar suas escolhas ou o que acontece com o seu corpo:

“... antes eu podia brincar bastante e agora menos, antes tinha menos riscos. Como quebrar o braço, agora se eu quebrar vou ter que amputar. Não foi normal, não deixei de brincar, mas brinco menos..." (S, 14 anos)

"... eu achava que com diabetes eu ia morrer, aí eu tirava nota baixa, mas depois eu soube que não tinha nada a ver, que poderia ter uma vida normal eu continuei fazendo atividade física, ir para a aula e estudar..." (V, 13 anos)

\section{O fardo da aplicação da insulina}

Segundo os depoimentos, a aplicação da insulina é considerada pelos adolescentes como um procedimento doloroso e incomodativo com dificuldades no transporte e que influencia o seu modo de vida. A aplicação de insulina é apontada como um dos principais fatores limitantes da doença para a manutenção de uma qualidade de vida:

\section{"... no começo eu tinha medo de agulha, porque na nossa família todo mundo tem, daí no começo foi diferente né, quem aplicava era minha mãe, ela queria aplicar e eu saía correndo, daí ela tinha que me segurar para fazer..." (AD, 14 anos)}

Quando questionados sobre o transporte da insulina, dois pontos principais apareceram na fala dos jovens: a responsabilidade de levar frascos tão sensíveis a outros lugares, impedindo momentos de lazer pelo medo de danificá-los. E, a necessidade de transportar o medicamento para os todos os lugares, ou ainda precisar voltar para casa para realizar a aplicação, atrapalhando atividades e lazer:

\footnotetext{
“... agora eu vou ter um passeio, a gente vai lá para o Beto Carrero, e vamos para um hotel fazenda ficar dois dias, daí eu não vou. Porque a minha mãe tem medo, por que tem muitos colegas, e é perigoso. Ela falou que às vezes eles podem quebrar os vidros da insulina e eu não vou ver....e daí o que eu vou fazer? Comprar vidro não tem como..." (AA, 16 anos)

“... a insulina a gente tem que ter muito cuidado, não pode balançar, não pode deixar cair, tem que estar sempre no gelo. E numa excursão dessa a responsabilidade é pra mim, vou ficar muito preocupada em cuidar da insulina, não sei se vou me divertir..." (AD, 14 anos)
}

Outras dificuldades de adesão à insulinoterapia diz respeito ao formato da seringa, quantidade necessária de material para realização as aplicações e desenvolvimento de habilidade para o manuseio:

... as primeiras vezes que eu tive que aplicar insulina, meu pai não deixou eu sair de casa, agora eu aprendi a aplicar sozinho, antes era só ele, e a primeira vez ele também não teve jeito e acabou me machucando. Eu ficava bravo, e a gente ficava brigando, né!? Daí, passaram alguns meses e comecei a olhar como ele fazia, e comecei eu mesmo a fazer. Uso uma seringa de manhã. A seringa do meio-dia eu lavo e uso de noite também. Faço isso para não gastar assim muita seringa, porque eu tenho que usar três por dia, entende? Daí eu uso só essas duas. Ganho de uma amiga diabética do meu pai uma seringa mais fina. Porque se for para comprar, minha família não tem condição. É no posto também, só que elas são grossas e grandonas, e essa dali que eu ganho pelo menos são mais fininhas, as outras são muito grossas..." (M,15 anos) 


\section{Livres para "voar": a caneta injetora de insulina e a autonomia}

Quando questionados sobre as diferenças entre a aplicação com seringa ou caneta, todos os adolescentes responderam que preferem a caneta porque é mais fácil de carregar, aplicar, menos dolorida, tem maior facilidade de armazenamento, maior controle da dosagem e é aplicável em qualquer ambiente, significando maior liberdade de ir e vir. Assim, aumenta sua autonomia e mostra para o grupo, profissionais de saúde e para sua família que ele pode ser responsável pelo seu corpo e seu tratamento:

“... a caneta. Porque ela é mais fácil de carregar, de aplicar e não precisa ficar guardando na geladeira..." (A, 18 anos)

"... eu escolheria a caneta porque a outra tinha que usar uma vez só a agulha, a caneta dá para usar 2 vezes, a seringa tinha que puxar a quantidade de insulina e a agulha era mais grossa, a caneta só precisa girar pra escolher a quantidade de insulina, é mais fácil..." (J, 17 anos)

“... a caneta, pelo fato de ser mais prático, vamos supor, eu posso aplicar em qualquer lugar, e pelo fato da agulhazinha ser menor é mais discreta e não necessita invadir a geladeira da casa de amigos e parentes para armazená-la..." (J, 17 anos)

"... eu posso fazer a insulina a hora que eu quiser, sem precisar pedir ajuda pra ninguém" (A,18 anos)

“... eu diria que é mais fácil para aplicar agora (com a caneta), as pessoas que olham de fora não vão achar que é outra coisa, tem muita gente que acha que a gente tá aplicando droga no corpo..." (A, 18 anos)

"... meus amigos acharam interessante, alguns falam que é legal, eu digo que não é tão legal assim, mas é bom ter a caneta porque eu e os meus amigos vamos jogar bola, daí eu passo mal e tenho que sair do jogo e ir pra casa fazer insulina. Agora não! Eu levo junto, aplico, espero 5 minutos e volto a jogar..." (V, 14 anos)

\section{DISCUSSÃO}

Diante dos resultados apontados nesta pesquisa os responsáveis, as equipes de saúde e o poder público têm um papel fundamental em apoiar os adolescentes com DM1 a desenvolverem habilidades para enfrentarem as dificuldades a que, inevitavelmente estão expostos, estimulando a apropriação de sua condição de saúde através da promoção ao autocuidado apoiado.

Observou-se que o concretismo, os exageros e as ideias radicais ainda estão presentes de maneira incisiva nos pensamentos dos adolescentes, característica típica deste ciclo e vida. A incorporação de novos hábitos alimentares e de vida, e a introdução de uma rotina terapêutica a ser seguida, acarretam consequências que podem ser mais difíceis de vivenciá-las e de serem trabalhadas na adolescência devido aos conflitos vivenciados durante este período, como a necessidade de independência e autonomia, valorização e senso de identidade de grupo, a ideia de indestrutibilidade, as alterações corporais da puberdade, a imaturidade e a reflexão que faz sobre si próprio e sobre a vida, bem como, seus valores e a desilusão do mundo adulto ${ }^{4,11}$.

As necessidades do adolescente estão associadas às questões de sua existência no mundo e à preocupação de sentir-se real, de buscar a sua própria identidade e o seu lugar no mundo. Nesse contexto, também se deve considerar que no tratamento da DM1 a dinâmica familiar, a relação médico-paciente, bem como, aspectos psicossociais e afetivos podem influenciar no controle do diabetes ${ }^{12}$. Além disto, o controle metabólico na adolescência tende a se deteriorar em função das mudanças hormonais típicas desta faixa etária. O declínio na produção de insulina associada a maior resistência insulínica e maiores riscos de hipoglicemia fazem com que o controle da taxa de glicose seja mais difícil ${ }^{13}$.

$\mathrm{Na}$ visão ampliada de saúde um adolescente com DM1 exerce vários papéis na sociedade e necessita que os profissionais de saúde o auxiliem no controle da sua condição com o mínimo de interferência na qualidade de vida deixando de enquadrá-lo no "grupo de diabéticos". Se a doença é biológica, consequentemente o estado patológico não pode ser chamado de anormal no sentido absoluto ${ }^{14}$. Nesta visão, a pessoa que descobre ter DM1 seria normal na sua situação de saúde, sendo assim, caberia aos profissionais, juntamente com os familiares, colegas e amigos, contribuir na construção de novos significados para a sua condição de saúde ${ }^{15}$.

Durante a adolescência, momentos de reunião com seu grupo e a ausência dos pais, são de extrema importância para a formação da personalidade e afirmação do seu espaço no mundo. O uso da insulina, muitas vezes, limita a circulação do adolescente em ambientes diferenciados em algumas ocasiões, como nas atividades escolares ou nas reuniões com os amigos que precisam ser canceladas, por exemplo, pelo fato de não ser possível o transporte ou armazenamento da insulina em outros espaços ${ }^{1,2}$.

Desde que a insulinoterapia é um aspecto essencial do tratamento o adolescente terá que se acostumar com as injeções diárias de insulina e os métodos invasivos de controle glicêmico que orientam a dose de insulina a ser aplicada. Os adolescentes associaram a seringa e a agulha ao medo e as dores bem como alguns referiram preconceito por parte dos colegas pela analogia com o uso de drogas ilícitas. A caneta pode auxiliar na adesão ao tratamento, pois reduz o trauma 
e a dor das aplicações, não precisa de assepsia, a aspiração da dose é adequada e não necessita de eventuais correções, como por exemplo, a presença de bolhas de ar na seringa. A dose é controlada e precisa e pode se autoadministrada por pessoas com pouco conhecimento ou prática ${ }^{15-17}$.

Todos os sujeitos da pesquisa utilizavam dois tipos de insulina, assim em cada horário de aplicação, geralmente antes das refeições, realizavam duas aplicações com a caneta. Quando a aplicação era feita com a seringa, as insulinas eram misturadas, perfazendo uma aplicação em cada horário. No entanto, mesmo realizando mais aplicações ao dia, a caneta foi considerada menos dolorosa, pois as agulhas são mais curtas e finas, reduzindo a sensação de dor.

A facilidade na utilização de canetas de insulina se encaixa em um estilo de vida agitado, característica do contexto social da atualidade, e pode melhorar o controle do DM1 com menos impacto na qualidade de vida de um adolescente. Assim, um estilo de vida com atividade social que cresce conforme a idade reflete diretamente o discurso mais numeroso dos adolescentes maduros sobre os benefícios do uso da caneta encontrados neste estudo. Santos \& Enumo ${ }^{18}$ relataram que uma das maneiras encontradas para estes jovens conviverem com o fato de estarem com uma doença crônica é "encarar" a realidade, por meio do enfrentamento. Tentam, dessa maneira, lidar com a situação, focalizando-se no problema, buscando cooperar, seja fazendo a aplicação da insulina ou seguindo a dieta. Além disso, adolescentes em estágio final usam mais estratégias de enfrentamento do que aqueles no início da adolescência, porque eles são mais propensos a tentar estratégias alternativas para resolver os problemas. E com a maturação cognitiva e a exposição a estressores provavelmente o adolescente em desenvolvimento torna-se mais capaz de atender, avaliar e escolher estratégias mais eficazes, um processo que pode incluir uma avaliação de resultados positivos da sua estratégia. Por outro lado, adolescentes mais jovens não se veem como responsáveis e sim receptores de ações e eventos ${ }^{19}$.

Esta estratégia de enfrentamento utilizada por adolescentes mais maduros faz com que a utilização da caneta auxilie na autonomia do tratamento. Nesse sentido, havendo disponibilidade de tecnologias que diminuem as dificuldades de uma doença crônica, seria prudente que o acesso a elas fosse facilitado pelos responsáveis, gestores e profissionais de saúde. Todos podem influenciar nas decisões, pois os processos produtivos nesse espaço só se realizam em ato e nas intercessões entre trabalhador e usuário. Os resultados desvelados nesse estudo mostram que a caneta injetora é uma tecnologia dura que encontrou ressonância no cuidado do diabetes na visão do adolescente. O uso melhorou a qualidade de vida e a circulação social pois possibilitou um posicionamento mais independente em relação às famílias e à rotina de vida, tornando-o protagonista de seus cuidados em saúde. Na perspectiva do cuidado centrado na pessoa, os resultados encontrados neste estudo sugerem que a inclusão da caneta injetora de insulina no rol de insumos previstos para o tratamento do DM1 contribuirá para uma melhora na qualidade de vida do adolescente demonstrando respeito às necessidades subjetivas e ao estímulo ao autocuidado.

\section{AGRADECIMENTOS}

Agradecemos ao Estado de Santa Catarina pela bolsa de estudo PIPe/artigo 170 e ao Laboratório Lilly pela doação de dispositivos caneta utilizados neste estudo.

\section{REFERÊNCIAS}

1. Fialho FA, Dias IMAV, Nascimento L, Motta PN, Pereira SG. Crianças e adolescentes com diabetes mellitus: cuidados/ implicações para a enfermagem. Rev Baiana Enferm. 2011; 2(2):145-54. http://dx.doi.org/10.18471/rbe.v25i2.4820

2. Santos RAB, Pinto SL, Almeida VCF, Lisboa KWSC, Marques SF. Diabetes mellitus tipo 1 : implicações na qualidade de vida dos adolescentes. Revista e-ciência. 2013;1(1):1-17.

3. Damião EBC, Dias VC, Fabri LRO. O adolescente e o diabetes: uma experiência de vida. Acta Paul Enferm. 2010;23(1):41-7. http://dx.doi.org/10.1590/S0103-21002010000100007

4. Schneider KLK, Martini JG. Cotidiano do adolescente com doença crônica. Texto Contexto Enferm. 2011;20(n. spe): 194-204. http://dx.doi.org/10.1590/S0104-070720110005 00025

5. Fonseca ALB, Tavares VS, Aquino AC, Mariano MSS, Silva EG. Reflexões sobre as repercussões da diabetes no comportamento adolescente. Psicol Foco. 2009;2(1):58-65.

6. Novato TS, Grossi SAA, Kimura M. Qualidade de vida e autoestima de adolescentes com diabetes mellitus. Acta Paul Enferm. 2008;21(4):562-67. http://dx.doi.org/10.1590/S010321002008000400005

7. Cavinatto SM. Doença crônica, autocuidado e autonomia: estudo de um processo educativo junto a pacientes com diabetes melito tipo 2 [dissertação]. Campinas: Universidade Estadual de Campinas; 2003. https://doi.org/10.20396/ccfenf12018 1496

8. Merhy EE, Baduy RS, Seixas CT, Almeida DES, Slomp Júnior H, organizadores. Avaliação compartilhada do cuidado em saúde: surpreendendo o instituído nas redes. Rio de Janeiro: Hexis; 2016.

9. Flick U. Uma introdução à pesquisa qualitativa. 3. ed. Porto Alegre: Artmed; 2009.

10. Bardin L. Análise de conteúdo. São Paulo: Edições 70; 2011. 
11. Moretto CC. Experiências com um grupo de adolescentes: um estudo psicanalítico [tese]. Campinas: Pontifícia Universidade Católica de Campinas; 2013. https://doi.org/10.17143/ciaed/ xxiilciaed.2017.00248

12. Minanni CA, Ferreria AB, Sant'Anna MJC, Coates V. Abordagem integral do adolescente com diabetes. Adolescência \& Saúde. 2010;7(1):45-52.

13. Polak $M$, Touraine $P$, editors. Transition of care: from childhood to adulthood in endocrinology, gynecology, and diabetes. London: Karger; 2018.

14. Canguilhem G. O normal e o patológico. Rio de Janeiro: Forense Universitária; 2002.

15. Ayres JRCM. O cuidado, os modos de ser (do) humano e as práticas de saúde. Saúde Soc. 2004;13(3):16-29. http://dx.doi. org/10.1590/S0104-12902004000300003

16. Maia FFR, Araujo LR. Uso da caneta injetora de insulina no tratamento do diabetes mellitus tipo 1. J Pediatr. 2002;78(3): 189-92. http://dx.doi.org/10.1590/S0021-75572002000300004

17. Campos RA. Insulinoterapia. Nascer e Crescer. 2011;20(3): 182-4.

18. Santos JR, Enumo SRF. Adolescentes com diabetes mellitus tipo 1: seu cotidiano e enfrentamento da doença. Psicol Reflex Crít. 2003;16(2):411-25. http://dx.doi.org/10.1590/S010279722003000200021

19. Williams K, McGillicuddy-De Lisi A. Coping strategies in adolescents. J Appl Dev Psychol. 2000;20(4):537-49. http:// dx.doi.org/10.1016/S0193-3973(99)00025-8

\section{Autoras:}

DEISI MARIA VARGAS

Médica. Doutorado em Pediatria, Programa de Pós-Graduação em Saúde Coletiva (PPGSC), Universidade Regional de Blumenau (FURB).

Orcid: http://orcid.org/0000-0003-4389-2670

E-mail: deisifurb@gmail.com

ANA LÚCIA BeRTARELLO ZeNI

Bióloga. Doutorado em Neurociências, PPGSC, Universidade Regional de Blumenau (FURB).

Orcid: http://orcid.org/0000-0002-9904-9861

E-mail: anazeni@furb.br

ADRIANA LOBO MULLER

Psicóloga. Mestre em Saúde Coletiva, PPGSC, Universidade Regional de Blumenau (FURB).

Orcid: http://orcid.org/0000-0002-9262-8422

E-mail: adrilmuller@hotmail.com

Claudia Regina lima Duarte da SiLVa

Enfermeira. Doutorado em Enfermagem, PPGSC, Universidade Regional de Blumenau (FURB).

Orcid: http://orcid.org/0000-0002-4813-1603

E-mail: claudiaduarte1108@gmail.com 